\title{
Einleitende Bemerkungen zum Thema des Dossiers: "Die deutsche Literatur und der Nobelpreis"*
}

Helmut Galle

In seinem vielzitierten Artikel „The Scandal of the Nobel Prize” von 1984 greift George Steiner den unverbesserlichen ,politischen, ästhetischen und psychologischen Konservatismus" "an, der bis dahin das Muster der Entscheidungen für, aber vor allem auch gegen Werke und Autoren bestimmt habe. Die Liste der von ihm ins Feld geführten Nicht-Prämierten ist lang und enthält - auch aus heutiger Sicht - fast ausschließlich Namen, die einem Kenner der Literatur des 20. Jahrhunderts preiswürdig erscheinen dürften, freilich auch dem Nichtkenner, denn es sind zwangsläufig Namen, die dem Kanon der Moderne eingeschrieben sind. Bemerkenswert ist auch, dass von den fünf Autoren, die man laut Steiner seinerzeit zu Recht als papabili handelte, in der Zwischenzeit vier ausgezeichnet wurden. ${ }^{2}$ Arbiträr

Redaktioneller Beitrag. Der Text versucht die „missing links” des Dossiers durch eine kurze Betrachtung der an deutschsprachige Autoren vergebenen Preise auszugleichen.

1 „There is, unfortunately, no reason to suppose that the awards to come will depart from the general pattern of political, esthetic and psychological conservatism, officiousness and deja vu' (STEINER 1984: 5). [Die Seitenzählung folgt des Einzelartikels, der als Download beim Archiv der New York Times online erhältlich ist.]

2 „If such distinguished figures as Octavio Paz, as Milan Kundera, as V.S. Naipaul [...], as Claude Simon are said to be papabili, if there is hope for that superbly 
kann die Auswahlpolitik der Schwedischen Akademie also nicht gewesen sein, eher voraussagbar, da die Autoren von unbestreitbarem internationalem Renommee tatsächlich im Laufe der Zeit geehrt wurden. Nicht geehrt hingegen wurden Leonardo Sciascia oder Thomas Bernhard, die Steiner in einem Atemzug mit Stendhal und Kafka nannte und für den Preis reklamierte, eine Verleihung aber für unwahrscheinlich hielt, weil sie weniger bekannt seien, als die vor.

Die Polemik zielt auf die Funktion des Nobelpreises. Alfred Nobels Testament hatte die fünf Preise für diejenigen vorgesehen, „die im verflossenen Jahr der Menschheit den größten Nutzen gebracht haben.”’3 Für den Literaturpreis war zusätzlich bestimmt worden, dass er dem gelten sollte, „der in der Literatur das Beste in idealistischer Richtung geschaffen hat." Nun lassen sich bahnbrechende Entdeckungen zum Wohle der Menschheit schon in den Naturwissenschaften kaum unmittelbar im Folgejahr erkennen. Im literarischen Bereich ist es zweifellos bereits schwer zu beantworten, inwiefern sie den Menschen „Nutzen bringt”. ${ }^{4}$ Der zeitliche Aspekt spielt aber hier sicher noch eine wichtigere Rolle bei der Ermittlung des „Besten”, da es sich ja nicht um Verkaufszahlen handeln kann, sondern um ein abgewogenes Urteil über die Qualität, das prinzipiell die gesamte globale Buchproduktion einzubeziehen hätte. Um ein solches Urteil im „Namen der Menschheit” zu fällen, muss sich die aus einem Dutzend Experten bestehende Schwedische Akademie zwangsläufig auf jene Filter stützen, die in den einzelnen Kulturen dazu führen, dass ein Werk und sein Autor literarische Anerkennung finden. Diese Prozesse aber brauchen vor allem Zeit.

Um es in der Terminologie von Pierre Bourdieu auszudrücken: Wer sich als Schriftsteller in den Kämpfen um die Positionierung im literarischen

talented, brave voice from South Africa, Nadine Gordimer, there would, at the same time be little use in drawing Stockholm's attention to some of the truly original, unworldly, little-heralded writers who will matter tomorrow." (STEINER 1984: 5).

3 Deutsche Übersetzung des Testaments zitiert nach http://www.nobelpreis.org.

$4 \quad$ Vgl. zu diesen prinzipiellen Dissonanzen von Testament und Entscheidungspraxis den Essay von Wolfgang KLEIN (1997). 
Feld national so nachhaltig behaupten konnte, dass er auch international fraglos anerkannt wird, der hat den Bereich der Avantgarde bereits verlassen und ist mit seinen Generationskollegen ins Mittelfeld der kanonisierten, ehemaligen Avantgarde aufgerückt, deren symbolisches Kapital sich bereits weitgehend in soziales und ökonomisches Kapital verwandelt hat (vgl. Bourdieu 1999: 256 ff.). Im Nobelpreis würde dieser Prozess der Konsekration seinen Höhepunkt finden, sowohl was die soziale Anerkennung als auch was die finanzielle Würdigung des literarischen Werts angeht. Die schwedische Jury wäre gewissermaßen ein Indikator für die histo-rischen Verschiebungen innerhalb des literarischen Feldes selbst und für dessen Verhältnis zur Gesellschaft im Ganzen.

Mit dem bedeutendsten und internationalsten aller Preise soll also, wie es scheint, ein Werk honoriert werden, das bedeutend genug ist, um in aller Welt durch die Ehrung empfohlen und gelesen zu werden. Wer in solcher Weise die Stimmen der Jury auf sich vereinigen kann, hat sich „durchgesetzt”, das heißt seine Bedeutung wird nicht mehr nur von ihm selbst behauptet, sondern auch von Kollegen nicht nur seiner eigenen Generation anerkannt. Es hat sich so sehr durchgesetzt, dass die nachdrängenden jungen Autoren seine nunmehr etablierten ästhetischen Prinzipien als ,veraltet” angreifen und ihre eigenen Konzepte dagegen setzen (die sich dann entweder allmählich selbst etablieren, oder marginalisiert werden). Der Name des bereits etablierten Autors ist zu diesem Zeitpunkt bereits ein Begriff auch außerhalb des Feldes der Literaturspezialisten und seine Art zu schreiben gilt als literarisch, zeitgemäß und ,herausragend” im Hinblick auf die gerade gültige Definition von Literatur. ${ }^{5}$ Solch eine Ausstrahlung über das literarische Feld hinaus gelingt Prosa-Autoren leichter als Lyrikern und Erzählern eher als Dramatikern. Für die internationale Verbreitung eines Autors ist daneben auch die Übersetzbarkeit eine wichtige Voraussetzung.

Ein Autor, auf den sich die Jury in Stockholm einigen kann, ist im Vorfeld bereits von den professionellen Kritikern und Autoren seines Landes

Bourdieu (1999: $353 \mathrm{ff}$ ) hebt hervor, dass mit dem personellen Wandel des Feldes stets auch der Kampf um die Definitionsmacht für „Literatur” verbunden ist, sich der Literaturbegriff folglich stets verändert. 
vorgeschlagen worden (oft mehrmals) und damit eher ein zustimmungsfähiger Kandidat als ein kontroverser Geheimtipp. Der Nobelpreis kann also - entgegen Steiners Kritik - in den allermeisten Fällen nur eine nachträgliche Bestätigung für einen Ruhm und eine Wirkung sein, die bereits sattsam registriert sind. Für Pascale CASANOva (2002: 193) beschränkt sich die Funktion der Jury, zumindest bis in die 60er Jahre des 20. Jahrhunderts hinein, auf ein letztinstanzliches Absegnen der bereits zuvor von „Paris”, der Hauptstadt der Res publica litterarum getroffenen Auswahlentscheidungen. Warum es in den letzten Jahren immer wieder zu Überraschungen kam, etwa als Dario Fo oder Elfriede Jelinek ausgezeichnet wurden, wird uns unten noch beschäftigen. Doch die Kritik ist in solchen Fällen eher noch heftiger als wenn ein allgemein zustimmungsfähiger Autor prämiert wird. Der von Steiner beklagte „Konservatismus” und die von Casanova verzeichnete „Nachträglichkeit” bei der Auswahl der Preisträger sind möglicherweise keine unveränderlichen Eigenschaften, sondern treten als Symptome der literatursoziologischen Konstellation auf, in der sich die Schwedische Akademie befindet und die dem historischen Wandel unterworfen ist wie die literarischen Felder (wäre hier bereits der Singular angezeigt?), in dem die Autoren agieren. Dies soll den Leitfaden der folgenden allgemeinen Bemerkungen zur Vergabe des Preises an deutschsprachige Autoren bilden, ohne damit das Thema auch nur entfernt ausschöpfen zu können oder zu wollen.

Die Jury entscheidet bekanntlich nicht auf der Basis eines Publikumsvotums wie beim Grand Prix d'Eurovision. Auch handelt es sich nicht (zumindest nie ausschließlich) um Aspekte des Proporzes hinsichtlich des Geschlechts, des Kontinents, des Kulturkreises oder der internationalen Politik, wie vielfach vermutet wird. Die Mitglieder der Akademie und das von ihr mit der Vorauswahl betraute Komitee verfügen über eigene literarische Kriterien, die zumeist an den Werken ihrer Nationalliteratur beziehungsweise am Kanon der Weltliteratur gebildet wurden, und zwar in einer historischen Phase, die bereits eine Generation zurückliegt, wenn sie in die Akademie aufrücken. Das muss nicht unbedingt heißen, dass ihr Geschmack „konservativ” ist, aber er repräsentiert wahrscheinlich in den meisten Fällen eher ein dreißig Jahre zurückliegendes Spektrum als die Gegenwartsliteratur. 
Kjell Espmark, selbst seit 1981 Mitglied der Schwedischen Akademie, hat ein aufschlussreiches Buch zur Geschichte der Nobelpreispolitik verfasst (ESPMARK 1988), in das seine persönliche Erfahrung und das Studium der Protokolle seit Beginn des Jahrhunderts eingegangen sind. Er gliedert die ersten achtzig Jahre in Phasen, die mit dem Einfluss von bestimmten Jury-Mitgliedern zusammenfallen und folglich unter der Perspektive des Generationenwechsels gesehen werden können. Für die Entscheidungen der ersten Jahre war beispielsweise mit Carl David von Wirsén eine Figur verantwortlich, dem alle jüngeren Tendenzen aufgrund moralischer und ästhetischer Erwägungen verhasst waren: Damit wurden die um 1900 bereits europaweit berühmten Repräsentanten von Naturalismus und Realismus zwar zum Teil vorgeschlagen, hatten aber keine Chance, die Mehrheit der Mitglieder auf sich zu vereinigen. So erklärt sich, dass ein Paul Heyse, der selbst zu seiner besten Zeit, die fast ein halbes Jahrhundert zurücklag, kein Neuerer gewesen war und den Thomas Mann - sicher kein literarischer Revolutionär - „einen fast unanständigen Epigonen” nannte, 1911 den Nobelpreis erhalten konnte. ${ }^{6}$ Die beiden vorangegangenen deutschsprachigen Preisträger waren Theodor Mommsen (1902), ein herausragender Historiker und Republikaner, und Rudolf Eucken, ein neoidealistischer Philosoph, der 1908 nur gewählt wurde, weil er zur Kompromisslösung anstelle der (noch) nicht mehrheitlich akzeptablen Hauptkandidaten Selma Lagerlöf und Algernon Swinburne wurde, obwohl selbst das Gutachten ihn nicht empfohlen hatte (vgl. Espmark 1988: 36). In diesen Anfangsjahren übte auch der Wortlaut des Testaments von Alfred Nobel noch eine gewisse Wirkung aus. Nobel hatte gefordert, dass das prämierte Werk eine „idealistische” Prägung haben sollte (EsPMARK 1988: 10). Eine solche weltanschauliche Einschränkung mochte die Wahl von Mommsen und Eucken noch erklären, spielte aber in den späteren Jahren gegenüber der literarischen Bewertung kaum noch eine Rolle.

Die Jury unter der Ägide von Wirsén (1900 bis 1911) folgte in ihren Entscheidungen einem Literaturbegriff, der eben durchaus von Kriterien bestimmt war, die kurz darauf von neuen Definitionen abgelöst wurden. Das zeigt sich in den Begründungen für die Preisträger ebenso wie in den

6 In einem Brief an Maximilian Harden, zit. nach HäNTZsCHEL 1990. 
Ablehnungen, die meist die erzählerische und dramatische Leistung anerkennen, dann aber an der im Werk zum Tragen kommenden Haltung der Autoren radikal Anstoß nehmen. Tolstoi wurde abgelehnt wegen seiner „fatalistischen Anschauungen” (25), Zola wegen des „Geistlosen, oft grob Zynischen in seinem Naturalismus” (27), Ibsen wegen der „Negativität und Rätselhaftigkeit" seines Spätwerks (28). An Gerhart Hauptmann moniert ein Gutachten von 1902, dieser habe ,sich in die widerlichsten Schilderungen von Trunksucht und Laster, das fast bis zur Blutschande getrieben wird, vertieft" (28).

Nachdem es 1911 zu einem ersten Generationenwechsel in der Jury gekommen war, konnten nun Autoren ausgezeichnet werden, die zuvor noch heftig für ihren Pessimismus und die Ästhetik des Hässlichen kritisiert worden waren. Gerhart Hauptmann, der bis heute wichtigste Exponent des deutschen Naturalismus, profitierte 1912 als Erster von dieser Verlagerung. Gleichwohl war der Expressionismus in der Zwischenzeit, wenn nicht abgelöst, so doch überlagert worden von den konkurrierenden Strömungen des „Stilpluralismus” der Jahrhundertwende: Symbolismus, Décadence, Impressionismus, Jugendstil, Neuromantik, Nervenkunst, um nur einige der geläufigen Etikettierungen zu nennen. Und nicht zuletzt waren im Jahr 1910 zwei emblematische Gedichte des Expressionismus erschienen: Georg Heyms „Gott der Stadt” und Jakob van Hoddis „Weltende”. Zweifellos gehört Gerhart Hauptmann zu diesem Zeitpunkt nicht mehr zur „Avantgarde”, auch wenn er selbst sich deutlich von seinen naturalistischen Anfängen entfernt hatte und noch über dreißig Jahre lang literarisch aktiv blieb. ${ }^{7}$ Er wird geehrt als Avantgardist einer - im schnell-

Es soll hier nicht der Eindruck erweckt werden, dass Hauptmann um 1911 kein fruchtbarer Autor mehr gewesen sei; ganz im Gegenteil hat er in vielerlei Hinsicht auch nach dem eigentlichen naturalistischen Jahrzehnt (1885-1895) eine bemerkenswerte Entwicklung durchgemacht, die ihrerseits die jeweils aktuellen Tendenzen aufgreift und auf die Zeitgenossen zurückwirkt. Die Vitalität des 60-jährigen Hauptmann hat schließlich noch ihr Portrait im Zauberberg gefunden. Andererseits gehen die eigentlichen ästhetischen Impulse nun von den nachwachsenden Kohorten aus: Um 1900 George, Rilke, Hofmannsthal, Wedekind und Thomas Mann, um 1910 die Expressionisten, ab 1920 die Neusachlichen. 
lebigen Literaturfeld ${ }^{8}$ - bereits zum Establishment avancierten Generation. ${ }^{9}$ Die Abkehr von naturalistischen Positionen hatte in den innerliterarischen Debatten Frankreichs und Deutschlands bereits in den frühen 90er Jahren des 19. Jahrhunderts begonnen (MEYER 2000: 75); die „Durchsetzung” des Naturalismus auf der Ebene der universalen Kanonisierung dauerte noch zwei Jahrzehnte: zu spät für den Hauptexponenten Zola, der bereits 1902 gestorben war.

Der für das Jahr 1919 geehrte Carl Spitteler war bereits 1914 Kandidat gewesen, damals aber aus politischen Rücksichten nicht gekürt worden: Seine betont pazifistische Haltung hätte bei Deutschen und Österreichern Zweifel an der Neutralität des Komitees ausgelöst (EsPMARK 1988: 44). Man schien in den mythologischen und universalistischen Tendenzen seines Werks eben jene Dimension des über die nationalistischen Querelen hinausweisenden Humanismus erkennen zu wollen, die Nobel zur Bedingung gemacht hatte. Spitteler ist laut Steiner eine der Ausnahmen, bei denen die Wahl der Akademie die Aufmerksamkeit des heutigen Publikums auf einen zu Unrecht vernachlässigten Autor lenken könnte. Die Bedeutung seines Werks für die Schweizer Literatur wird von Isabel Hernandez im Dossier dieses Heftes herausgearbeitet.

Thomas Mann war Espmark zufolge bereits 1924 im Gespräch, konnte sich aber damals nicht durchsetzen, weil man sich über die Bedeutung der Buddenbrooks nicht im Klaren war. ${ }^{10}$ Als er 1929 dann gerade für dieses Buch ausgezeichnet wird, zeigt sich erneut der Verzögerungseffekt, der über

8 In Frankreich verlief die „Ablösung” des Naturalismus durch Symbolismus und Décadence um einige Jahre früher und bedeutend heftiger, wie Pierre BOURDIEU (1999: $202 \mathrm{ff}$ ) in der Beschreibung des literarischen Feldes Paris zeigt.

9 Dass Hauptmann in der politischen Sphäre noch nicht ganz etabliert war, hängt mit der Affinität der naturalistischen Ästhetik zum Sozialismus und der reaktionären Haltung von Wilhelm II. zusammen.

10 Neben den großen Romanen haben zweifellos auch Erzählungen wie „Der Tod in Venedig" zum Ruhm Thomas Manns beigetragen. Nach Hans R. VAGET (2005: 581) wurde der Autor mit diesem Werk zur internationalen Größe mit dem „Nimbus des Klassikers”: „Diese Arbeit erst machte ihn zum Nobelpreis-Kandidaten, wenn auch noch nicht in den Augen der schwedischen Akademie, so doch nach dem Dafürhalten der internationalen literarischen Öffentlichkeit.” 
den Ent-scheidungen der Jury waltet. Zwar ist man sich nun über den literarischen Wert des Frühwerks einig, das noch ganz im Geist der realistischen Erzählkunst des 19. Jahrhunderts steht (bezeichnenderweise nun mit Tolstoi verglichen, der um die Jahrhundertwende noch mit den Moralvorstellungen des damaligen Komitees kollidierte), lehnt aber den Zauberberg ab, der, wie es in einem Gutachten von 1928 hieß, „ein außerordentliches Werk von bedeutendem Gehalt [ist], jedoch vom ästhetischen Standpunkt aus zu breit und schwerfällig, als daß es zu Manns besten Leistungen gerechnet werden könnte" (Espmark 1988: 68). Wenn man diesen Roman heute als das ambitionierteste Erzählprojekt von Thomas Mann einschätzt, das sich neben Musil und Döblin behaupten kann ${ }^{11}$, und ästhetisch am ehesten auf der Höhe seiner experimentierfreudigen Zeit ist, so zeigt die Entscheidung der Jury, dass ihr diese Qualitäten vier Jahre nach Erscheinen des Romans noch nicht erkennbar waren. Auch Musil und Döblin haben den Preis ja nicht erhalten, ebenso wenig wie Franz Kafka, Hermann Broch, Marcel Proust, Virginia Woolf und James Joyce. Alle gelten heute als repräsentativ für die „klassische Moderne”, starben aber zu früh, als dass sie noch zu Lebzeiten die Stockholmer Konsekration hätten erleben können. Für Kafka findet der Artikel von Celeste Ribeiro de Sousa weitere Gründe. $^{12}$

Erst mit Ende des Zweiten Weltkrieges setzt Espmark einen Paradigmenwechsel in der Schwedischen Akademie an, die 1948 T. S. Eliot „für seine außerordentliche Leistung als Bahnbrecher der modernen Dichtung" ehrt. Eliot ist zwar nur sieben Jahre jünger als Joyce und Woolf, überlebte diese aber um mehr als zwanzig Jahre. Zu einer Zeit, da die großen Experimente der ersten Jahrhunderthälfte bereits Geschichte sind und ihre Bedeutung für die Nachgeborenen kaum noch angezweifelt werden kann, etabliert sich in der Begründungspraxis das Konzept des „Bahnbrechers”, also des Avantgardisten, um einem Literaturkonzept Rechnung zu tragen, das eben durch Figuren wie Joyce und Kafka erst geprägt wurde. Die nach

11 Vgl. etwa LehNert 2005: $153 \mathrm{f}$.

12 Kafkas erste Publikation datiert von 1908. Er starb 1924 mit nicht einmal 41 Jahren. Das durchschnittliche Alter der in diesem Zeitraum prämierten Autoren liegt bei 59 . 
1945 für preiswürdig erachtete Literatur kann nun durchaus den Charakter des Hermetischen tragen, wie die Poesie Paul Valérys, der den Preis nur durch seinen Tod im selben Jahr verfehlte. In jedem Fall sieht die Jury in Hermann Hesse, André Gide, T. S. Eliot und William Faulkner Exponenten der Moderne, die zuvor ausgeschlossen waren, nun aber zu Vorbildern aufrücken. Man mag sich darüber streiten, in welchem Grade Hesse - im Vergleich mit 1947 noch lebenden Autoren wie Broch und Döblin - tatsächlich Epoche gemacht hat. Aber immerhin wurde er damals von manchen so eingeschätzt, wie sich an Thomas Manns Bemerkung ablesen lässt, der Steppenwolf stehe dem Ulysses und den Faux Monnaieurs ,an experimenteller Gewagtheit" nicht nach. ${ }^{13}$ Hesse war seit seinem Peter Camenzind (1904) ein erfolgreicher und äußerst produktiver Autor und hatte 1943 mit dem Glasperlenspiel noch einmal einen großen, eher visionären als modernistischen Entwurf zur Deutung des menschlichen Lebens vorgelegt, der in den ersten Nachkriegsjahren große Beachtung fand. ${ }^{14}$ Auch Hesse befindet sich zum Zeitpunkt der Preisverleihung ${ }^{15}$ in jedem Fall bereits im kanonischen Bereich des literarischen Feldes, gegen das sich die Generation der Gruppe 47 abzusetzen hatte. Er gehört bis heute zu den meistgelesenen deutschsprachigen Autoren weltweit.

Vergleicht man die Lebensdaten Hesses (1877-1962) mit denen Bertolt Brechts (1898-1956), der immer wieder als ein zentrales Versäumnis bei der Preisvergabe genannt wird, so wird deutlich, dass dieser erst zwanzig Jahre später ins Leben und in die Literatur eingetreten ist. Wurde mit Hesse ein Werk geehrt, das von Anfang an gegen institutionelle Macht und die zerstörerischen Tendenzen der Moderne gesetzt war und im Schatten der Nazi-Diktatur gestanden hatte, so war Brecht zwar schon in den 20er Jahren

13 In der Einleitung zu einer amerikanischen Demian-Ausgabe (zitiert nach ScHWARZ 1993: 132).

1435 Auflagen bis 1949 (vgl. BARNER 1994: 33).

15 Laut Preisrede erhielt er die Auszeichnung auch eher ,für seine inspirierte Verfasserschaft, die in ihrer Entwicklung neben Kühnheit und Tiefe zugleich klassische Humanitätsideale und hohe Stilwerte vertritt". Die Zitate aus den Preisreden stammen aus der „Liste der Nobelpreisträger für Literatur” bei Wikipedia. 
ein erfolgreicher Theaterautor; seine wichtigsten Stücke entstanden jedoch im Exil und die anschließende Option für den sozialistischen deutschen Staat markierte ihn in der politisch aufgeladenen Atmosphäre der Nachkriegsjahre - nicht zu Unrecht - als ,kommunistischen Autor”. ${ }^{16}$ Seine breite internationale Anerkennung als Pionier der modernen Dramatik setzte erst in den 50er Jahren des 20. Jahrhunderts ein und bekam einen endgültigen Schub durch die Politisierung der westlichen Literatur in den 60er Jahren. Bei seinem Tod war seine Rolle für das moderne Drama, die ihn in den Augen der Akademie als „Bahnbrecher” empfohlen hätte, noch nicht so allgemein durchgesetzt wie in den folgenden Jahrzehnten. Tatsächlich wurde er überhaupt erstmals „1956 von schweizerischer (!) Seite” vorgeschlagen und starb noch im selben Jahr. ${ }^{17}$

Das Kriterium der avantgardistischen Schreibweise behält von nun an seine Gültigkeit und rechtfertigt später die Wahl von Autoren wie SaintJohn Perse und Samuel Beckett, aber auch Entscheidungen, die zum Teil einem kulturellen Proporz geschuldet scheinen wie die Preisverleihungen an Nelly Sachs (1966) und Miguel Asturias. Espmark sieht im Preis für die deutsche Jüdin im schwedischen Exil auch eine verspätete Wiedergutmachung für die versäumte Würdigung der deutschen Expressionisten, wie etwa Else Lasker-Schülers, zu deren Dichtung die Aussage der Preisrede auf Nelly Sachs ebenso gut passen würde: ,dass ihre Symbolsprache 'modernistische Kühnheit in der Eingebung mit dem Echo uralter biblischer Poesie vereinigt',, (Espmark 1988: 203). Auf Nelly Sachs geht der Artikel von Helmut Galle ein.

Paul Celan, der Nelly Sachs persönlich, thematisch und ästhetisch nahesteht, wird in dem Beitrag von Juliana P. Perez behandelt. Sein Werk wurde von Steiner als die ,tiefste, innovativste lyrische Dichtung der westlichen Literatur in unserer Zeit"18 bezeichnet und damit postum für

16 Man bedenke die öffentliche Wirkung seines Schweigens zum 17. Juni 1953 sowie den Staatspreis der DDR 1951 und den Stalin-Friedenspreis 1954.

17 Vgl. Fritz Pauls (das Ausrufezeichen ist von ihm) Nachwort zu Espmarks Buch (Paul 1988: 215).

$18,[\ldots .$.$] the profoundest, the most innovative lyric poetry in western literature of$ our time" (STEINER 1984: 1). 
absolut preiswürdig erklärt. Auch im Fall von Celan sollte man beachten, dass er nicht nur verhältnismäßig jung starb (geboren 1920 in Czernowitz, beging er vermutlich am 26. 4. 1970 in der Seine Selbstmord). Er dürfte zu Lebzeiten nicht für den Preis vorgeschlagen worden sein, und die Rezeption seiner Publikationen in den 60er Jahren durch die Kritik war zwiespältig. Zusätzlich belasteten seine Position in der Öffentlichkeit die ebenso haltlosen wie hartnäckigen Plagiatsvorwürfe, die Claire Goll gegen ihn vorgebracht hatte. Während Paul Celans Werk heute tiefgründiger, vielschichtiger und radikaler erscheint als die Lyrik Nelly Sachs', musste es damals als wesentlich hermetischer, zur Unverständlichkeit tendierend gelten. Die Bemühungen um die Interpretation von Paul Celans Dichtung haben seit den 70er Jahren - und weit über die Literaturwissenschaft hinaus - in einem Maße zugenommen, die am ehesten mit dem Phänomen Kafka zu vergleichen ist. Zwar sicherte ihm ein Text wie die „Todesfuge” schon sehr früh einen festen Platz im Kanon der Literatur nach und über Auschwitz, doch wäre seine gegenwärtige Position in der Weltliteratur ohne die konstanten Anstrengungen von Interpreten und Übersetzern kaum vorstellbar.

Im Jahr 1972 erhielt Heinrich Böll (1917-1985) den Nobelpreis „,für eine Dichtung, die durch ihre Verbindung von zeitgeschichtlichem Weitblick und liebevoller Gestaltungskraft erneuernd in der deutschen Literatur gewirkt hat". Böll war zweifellos die international herausragende Figur der Literatur der Nachkriegszeit. Nach den Verheerungen durch Ideologie und Krieg kann jene Rückgewinnung realistischer und humanistischer Positionen, die hier zu Recht als „Erneuerung” bezeichnet werden, zu einem nicht geringen Teil als Verdienst Bölls angesehen werden, auch wenn er keineswegs der einzige Vertreter jener Bewegung war, die meist mit der Gruppe 47 identifiziert wird. Böll ist vor allem als politischer denkender und handelnder Mensch zu der Figur geworden, die Deutschland seit den 50er Jahren wieder zu einem gewissen Ansehen verholfen hat. EsPMARK (1988: 157) hebt hervor, dass seit Thomas Mann, also 43 Jahre lang, ,kein Literaturpreis mehr nach Deutschland gegangen" war. Die Preisverleihung fällt in eine Zeit, in der Bölls kritische Haltungen auch gesamtgesellschaftlich zu dominieren begannen: Die sozialliberale Koalition unter Willy Brandt hatte sich erfolgreich für die Aussöhnung mit der Sowjetunion und Polen eingesetzt und die Entspannung zwischen den Machtblöcken eingeleitet. Auch wenn 
die Akademie mit der Wahl Bölls nicht in die innenpolitische Auseinandersetzung Deutschland eingreifen wollte, wie von konservativer Seite geargwöhnt wurde (ESPMARK 1988: 136), hat man ihn zu einem Zeitpunkt bedacht, als seine Position im Kanon (bis hin zur obligatorischen Schullektüre) längst fest verankert und selbst seine frühere politische Dissidenz zum Mainstream geworden war. Dass er sich vor seinem Tod noch einmal radikalisierte und seine Autorität für die Friedensbewegung und die in den Terrorismus abdriftende studentische Linke einsetzte, zeigt die Konsequenz seiner Haltung, ändert aber nichts mehr an der literarischen Einordnung seines Werks. Die ihm von der Jury attestierte „Erneuerung” betrifft weniger die ästhetischen Mittel als ethische Legi-timität der Literatur. Wenn hier die politisch-moralischen die ästhetischen Kriterien in den Hintergrund zu drängen scheinen, so entspricht dies durchaus dem in den 60er Jahren einsetzenden Trend, den sich die Akademie nun zu eigen machte, als er in den innerliterarischen Diskursen Deutschlands bereits abzuklingen begann.

Als britischer Staatsbürger in Zürich lebend erhielt Elias Canetti den Preis 1981. Canetti war 1905 in einer Familie sephardischer Juden in Bulgarien geboren und schrieb in einem Idiom, das nicht seine Muttersprache war. Irene Aron geht dieser spezifischen Situation in ihrem Artikel nach. Seit 1977 hatte sich eine neue Strategie bei der Verleihung durchgesetzt: Man wollte nun auf Autoren hinweisen, die international nicht dieselbe Wertschätzung genossen wie in ihrer jeweiligen Kultur. Canetti gehörte laut Espmark (1988: 113 f.) mit Isaac Bashevis Singer, Odysseas Elytis und Czesław Miłosz in die Reihe solcher Autoren. Alle drei sind weit über sechzig Jahre alt, sie sind Repräsentanten „kleiner Literaturen”, aber kaum international bekannt. Canetti, der vor allem mit seiner seit 1977 erscheinenden Autobiographie auf sich aufmerksam gemacht hatte, gehörte genau genommen gar keiner national verankerten Kultur an und war eben deshalb ohne das großräumige Publikum geblieben, das seine brillante Darstellung des Europa vor und zwischen den Kriegen verdiente. Hier ist vielleicht durch die Politik des Komitees eine Rezeption eingeleitet und verstärkt worden, die bis heute anhält.

Kurz vor Ende des Jahrhunderts wurde Günter Grass (1999) die Nobelwürde verliehen. Im Artikel von Marcus Mazzari findet sich die 
eingehende Würdigung seiner Werke seit den 50er Jahren, die seine beherrschende Stellung als international renommiertester deutscher Schriftsteller begründen. Bei dieser Entscheidung mag mitgespielt haben, dass Grass im Hinblick auf die literarische Eigenständigkeit wahrscheinlich von den meisten Akademiemitgliedern über Böll gestellt wurde, dem aber 27 Jahre zuvor als dem Älteren und dem Pionier der „Kahlschlagliteratur” der Vorrang gebührte. ${ }^{19}$ Seit Bölls Tod konnte Grass' Stellung nicht mehr angefochten werden, zumal er weiterhin beharrlich publizierte und in der öffentlichen Diskussion präsent blieb. Seine seit fünfzig Jahren anhaltende Bedeutung und sein Einfluss nicht nur auf deutsche Autoren legten die offizielle Bestätigung durch das Nobelpreiskomitee nahe. Nach seinem Bekenntnis, der Waffen-SS angehört zu haben, sind vereinzelt Stimmen aufgekommen, die die Rückgabe des Preises forderten. Dies scheint ungerechtfertigt, wenn der Preis für die literarische Leistung vergeben wurde, denn die Wirkung, die seit Erscheinen der Blechtrommel von diesem Werk ausging, wäre zweifellos eine andere gewesen, hätte der Autor zum damaligen Zeitpunkt dieses biographische Detail nicht verschwiegen. Ungeschehen machen lässt sich diese historische Wirkung im Jahr 2006 nicht mehr.

Die überraschendste Entscheidung des Nobel-Komitees war dann zweifellos der 2004 an die 1946 in Mürzzuschlag geborene Autorin Elfriede Jelinek vergebene Preis. Zwar war sie die erste Repräsentantin Österreichs, das im Verhältnis zu seiner kleinen Bevölkerung erstaunlich viel literarische Begabungen hervorgebracht hat und dem daher schon lange eine Auszeichnung ,zugestanden” hätte. Doch wäre der Preis - mit George Steiner - wohl eher für Thomas Bernhard erwartet worden, der freilich 1989 verstorben war. Jelinek publiziert seit den 70er Jahren und ihre Dramen gehören zu den meistgespielten zeitgenössischen Stücken in Deutschland.

19 Steiner (1984: 3) ignorierte solche Aspekte und sah in der Wahl Bölls lediglich das „Mittelfeld urban-liberaler Wohlanständigkeit” bestätigt, während Grass für ihn der „,bei weitem bessere Schriftsteller” sei. Aus heutiger Sicht erscheint freilich äußerst fraglich, welcher der beiden in politischer Hinsicht radikaler agierte. („The thoroughly embarassing preference of Heinrich Böll in 1972 over that far greater writer Günter Grass was wholly typical of the Swedish Adacamy's bias towards the middle ground of urbane and liberal decencies." STEINER 1984: 3). 
Typisch für sie ist die Thematik der Geschlechter- und Machtordnung. Ihr Roman Die Klavierspielerin wurde verfilmt und sie erhielt diverse nationale Literaturpreise. ${ }^{20}$ Gleichwohl ist sie eine äußerst umstrittene Autorin, deren politische Äußerungen in der außerliterarischen Öffentlichkeit ebenso Anstoß erregen, wie ihre Texte bei der Literaturkritik auf geteilte Meinungen stoßen. Als ästhetisches Verfahren reklamiert Jelinek das Prinzip der Montage: Die offenkundige - und vielfach monierte - Trivialität ihrer Texte sei Eigenschaft der öffentlichen und privaten Diskurse, die von ihr lediglich „in Szene” gesetzt würden. So wird sie in der Begründung der Jury denn auch geehrt „für den musikalischen Fluss von Stimmen und Gegenstimmen in Romanen und Dramen, die mit einzigartiger sprachlicher Leidenschaft die Absurdität und zwingende Macht der sozialen Klischees enthüllen”.

Hier zeichnet sich erneut eine Strategie ab, die ein verändertes Literaturverständnis zugrunde legt: Nicht die gesicherte Position im Kanon, nicht der anerkannte, konsekrierte Avantgardismus, nicht die Repräsentativität für eine Kultur und nicht die erwiesene Bedeutung im Rahmen einer kleinen Literatur werden hier gewürdigt, sondern eine durchaus exzentrische Position im literarischen Feld. Dass das Nobelkomitee dieses Risiko eingeht, hat inneren Dissens und Kritik von außen hervorgerufen, zeigt aber auch, dass für die Gegenwart mit einem Literaturbegriff zu rechnen ist, der gerade exzentrische Positionen als repräsentativ einstuft, wenn diese sich konsequent der Vereinnahmung durch die außerliterarischen Diskurse verweigert. Dies könnte auch eine Reaktion auf die Tendenzen der nachwachsenden „Avantgarde” (gemeint ist natürlich die „neue deutsche Popliteratur”) sein, die sich zwar im Kontrast zur „Hochliteratur” des arrivierten Feldes definiert, aber nicht ein besonderes symbolisches Kapital gegen den kommerziellen Erfolg der Etablierten ins Feld führt, sondern im Gegenteil die Konsumierbarkeit ihrer Texte betont und sich auch unmittelbar auf dem Markt behaupten kann, da ihre Marketingstrategien auf ein ähnlich gelagertes Interesse bei den Massenmedien stoßen.

Es bleibt abzuwarten, ob die Jury auch diese Entwicklung - mit der allfälligen Verzögerung - eines Tages aufgreifen wird oder ob dies eine

20 Unter anderem: Preis der Stadt Wien 1989, Bremer Literaturpreis 1996, BüchnerPreis 1998. 
periphere Erscheinung bleibt. Von einer gründlicheren Untersuchung der Komiteepolitik in den verschiedenen historischen Phasen darf man sich allerdings noch manche Einsicht in die Mechanismen der Formierung des literarischen Feldes auf nationaler und internationaler Ebene erwarten.

\section{Literaturverzeichnis:}

http://de.wikipedia.org/

http://www.nobelpreis.org/

BARNER, Wilfried (Hg.). Geschichte der deutschen Literatur von 1945 bis zur Gegenwart. Vol. XII. München, Beck 1994.

Bourdieu, Pierre. Die Regeln der Kunst. Genese und Struktur des literarischen Feldes. Übers. Schwibs, Bernd / Russer, Achim. Frankfurt a. M., Suhrkamp 1999. (Les règles de l'art. Genèse et structure du champ littéraire. 1992)

Casanova, Pascale. A República Mundial das Letras. Trans. Appenzeller, Marina. São Paulo, Estação Liberdade 2002. (La République mondiale des Lettres. 1999)

Espmark, Kjell. Der Nobelpreis für Literatur. Prinzipien und Bewertungen binter den Entscheidungen. Trans. Paul, Fritz / Volz, Ruprecht. Göttingen, Vandenhoeck \& Ruprecht 1988. (Det litterära Nobelpriset. Principer och värderingar bakom beslu-ten. 1986)

HÄNtzschel, Hiltrud. „Heyse, Paul”. Artikel in: W. KILLY (Hg.) Literaturlexikon. Band 5. Gütersloh / München, Bertelsmann 1990. 306 f.

KLEIN, Wolfgang. „Nobels Vermächtnis, oder die Wandlungen des Idealischen.” In: LiLi. Zeitschrift für Linguistik und Literaturwissenschaft. Heft 107. (1997)

http://lili.avmz.uni-siegen.de/ausgaben/lili202fr.html Zugriff am 28. 11. 2006.

LEHNERT, Herbert. „Thomas Mann und die deutsche Literatur seiner Zeit.” Thomas-Mann-Handbuch. Ed. Koopmann, Helmut. Frankfurt a. M., Fischer 2005. 137-163.

MEYER, Theo. „Das naturalistische Drama.” Naturalismus. Fin de siècle. Expressionismus. 1890-1918. Ed. Mix, York-Gothart. Vol. 7. Hansers Sozialgeschichte der deutschen Literatur vom 16. Jahrhundert bis zur Gegenwart. München, Hanser/dtv 2000. 64-76. 
๑ PAul, Fritz. „Kulturelles Gedächtnis und literarische Innovation. Ein Nachwort." Der Nobelpreis für Literatur. Ed. Espmark, Kjell. Göttingen, Vandenhoeck \& Ruprecht 1988. 206-216.

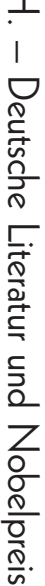

SCHWARz, Egon. „Hermann Hesse: Der Steppenwolf.” Romane des 20. Jahrbunderts. Interpretationen. Vol. 1. Stuttgart, Reclam 1993. 128-157.

SteIner, George. „The Scandal of the Nobel Prize.” New York Times Review of Books 30. 09. 1984. [Download aus dem Archiv der Zeitung unter: http://www.nytimes.com/]

Vaget, Hans Rudolf. "Die Erzählungen”. Thomas-Mann-Handbuch. Ed. Koopmann, Helmut. Frankfurt a. M., Fischer 2005. 534-610. 